Communications in Physics, Vol. 24, No.3S2 (2014), pp. 45-51

DOI:10.15625/0868-3166/24/3S2/5051

\title{
TIME RESOLVED TDLAS INVESTIGATION OF PRE-IONIZATION EFFECT IN DUAL HIPIMS DISCHARGES
}

\author{
NGUYEN VAN TAN AND DO HOANG TUNG \\ Institute of Physics, Vietnam Academy of Science and Technology \\ RAINER HIPLER \\ Institute for Physics, University Greifswald, Felix-Hausdorff Strasse 617489 Greifswald, \\ Germany \\ E-mail: dhtung@iop.vast.ac.vn
}

Received 20 June 2014

Accepted for publication 20 August 2014

\begin{abstract}
Time-resolved measurements have been performed during dual High Power Impulse Magnetron Sputtering (dual-HiPIMS) with two cathodes in a closed magnetic field configuration. The dual-HiPIMS system, operated at a repetition frequency $f=100 \mathrm{~Hz}$ and duty cycle of 1\%, was equipped with two different metallic targets (Ti, Cu). Preionization effect of the Ti pulse on the Cu pulse was investigated through argon excited atom density and temperature by means of tunable diode laser absorption spectroscopy. We observed an enhancement of metastable density due to pre-ionization effect. It is shown that the peak density in the Cu pulse increases about $30 \%$ for the case of $15 \mu$ s delay then decrease as the delay was prolonged. The effect displays even stronger in the peak density in the afterglow, as well as in the atom temperature behavior. Although metastable atoms are abundantly available in post-discharge, they play minor role in the ignition of the subsequence pulse.
\end{abstract}

Keywords: HiPIMS; time resolved TDLAS; metastable, pre-ionization effect.

\section{INTRODUCTION}

Magnetron sputtering (MS) deposition is a plasma-based coating technique well known [1, 2] since 1980. Inert gas ions (usually $\mathrm{Ar}^{+}$) are accelerated to bombard cathode/target made from the material to be deposited. Sputtered target atoms condensate onto a substrate and form a film. Growth and formation of the film are strongly influenced by ion and energy fluxes to the substrate $[3,4]$. High ionization degree of sputtered particles and high ion fluxes can be achieved in so-called high power impulse MS (HiPIMS) discharges [5] working at low frequency $(f \sim 100 \mathrm{~Hz})$ and short pulse width $\left(T_{a} \approx 10-100 \mu \mathrm{s}\right)$. HiPIMS discharges have high power pulse density (approximately kilowatt per centimeter square) and high concentration of charged particles [6] (typically $\geq 10^{18} \mathrm{~m}^{-3}$ ).

However, deposition rate in HiPIMS is rather low due to its high ionization degree of sputtered particles [7]. This is one of the drawbacks for wide application of HiPIMS in the coating industry. Many attempts have been made to overcome this limitation. One of those is the HiPIMS

(C)2014 Vietnam Academy of Science and Technology 
discharges working with higher repetition frequency $(f \geq 1 \mathrm{kHz})$ and longer pulse widths $\left(T_{a} \approx 100\right.$ $-400 \mu \mathrm{s}$ ) [8,9]. In such configuration the HiPIMS advantages are preserved and in addition the higher deposition rate $(\sim 2 \mu \mathrm{m} / \mathrm{min})$, which cannot be achieved by a conventional dc MS due to a target overheating, have been observed. Other efficient sputtering systems employing two magnetrons equipped with the same target material and synchronously applying the same cathode voltage have been developed in the last years, too. The dual-HiPIMS with open magnetic field, employing two identical target materials [10] and the so-called dual-MS with closed magnetic field (two magnetrons with opposite polarity of magnetic field ) [11,12]. Recently, a more flexible system is proposed by Stranak et al. and co-workers ${ }^{13}$ using a dual magnetron systems with closed magnetic field working at repetition frequency $f=100 \mathrm{~Hz}$ and pulse width $T_{a}=100 \mu \mathrm{s}$. In this system, the sputtering sources were equipped with different target materials $(\mathrm{Cu}, \mathrm{Ti})$ and driven at different cathode voltages to optimize the thin film stoichiometry. The delay between subsequent pulses, i.e. the delay between a Ti pulses followed by a $\mathrm{Cu}$ pulse, can also be varied to control the parameters responsible for thin film growth.

The influence of time delay on plasma behavior in a dual HiPIMS system was first studied in [13]. It was noted that the pre-ionization effect promotes the ignition of the subsequent pulse which results in a higher plasma density and smaller electron temperature. The role of metastable atoms in noble gas discharges have fundamental importance from the point of view of discharge sustaining, kinetics of excited species and influence on the electron distribution function. In gas mixtures, the collisions with metastables cause large range of reactions, including Penning ionization, stepwise ionization and chemical transformations. Metastable are abundantly available in post-discharge [14] and may contribute also to the fast ignition of the second pulse due to its low ionization energy.

Tunable diode laser absorption spectroscopy (TDLAS) has shown capability to diagnose time resolvedly both buffer gas metastable atom density and temperature $[14,15]$ as well as sputtered atom [14] in HiPIMS discharges. Therefore, it is worthy using time resolved TDLAS to investigate pre-ionization effect in dual HiPIMS discharges.

\section{EXPERIMENT AND DIAGNOSTIC METHOD}

\section{II.1. Experimental setup}

The experimental set-up has been described in detail elsewhere [16]. It consists of a vacuum chamber pumped by a turbomolecular pump to a base pressure of better than $10^{-5} \mathrm{~Pa}$. A dual-HiPIMS system with closed magnetic field has been developed for deposition of Ti-Cu films. Two commercial planar magnetrons (Gencoa, Liverpool, UK), with opposite magnetic field configurations, are attached to the upper flange of the vacuum chamber and located $11 \mathrm{~cm}$ below the flange. The magnetrons are equipped with $\mathrm{Ti}$ and $\mathrm{Cu}$ targets $50 \mathrm{~mm}$ in diameter. The working pressure is adjusted by a throttle valve installed between the vacuum chamber and the pump. All experiments were carried out in an argon atmosphere at a pressure $p=3 \mathrm{~Pa}$; the argon flow rate of $20 \mathrm{sccm}$ was controlled by a mass flow controller.

The high power electrical circuit of the dual sputtering system is based on a parallel combination of two identical pulsed power switches as employed in our previous works $[13,17,18]$. Each of the 2 electric loops employs a power switch in combination with a commercial direct current power supply (AE Pinnacle 3000) with a range down to $-1000 \mathrm{~V}$ designed for continuous 
operation and used to charge a large capacitor during the idle part of the pulses. A ballast resistor $(R=2.1 \Omega)$ serving without magnetron discharge as a dummy load was inserted in series with the magnetron cathode path. A home-built signal wave-form generator, employed to set repetition frequency $f$, pulse width $T_{a}$, and delay between subsequent pulses, was employed for activation of the power switches.

Measurements with different delays of $\mathrm{Cu}$ pulse from the Ti pulse were carried out. In order to magnify the pre-ionization effect,mean discharge currents $I_{\mathrm{Ti}}$ was set at $400 \mathrm{~mA}$ and $I_{\mathrm{Cu}}$ was set four time smallerat $100 \mathrm{~mA}$.

\section{II.2. Time resolved TDLAS}

Time-resolved light absorption spectroscopy was performed with the help of a tunable diode laser. The single mode diode laser (Toptica DL100) is equipped with an external cavity. The spectral band width is much smaller than the width the absorption profile in a low pressure glow discharge. The laser light beam is split into two parts with a beam splitter. The first beam is guided to a confocal Fabry-Perot interferometer with $1 \mathrm{GHz}$ free spectral range and detected with the first photo-diode to perform a calibration of the laser frequency scan. The second beam is launched into the discharge chamber where it passes parallel to the target and is detected on the opposite side of the chamber with the second photodiode. Measurements were carried out at a distance of $10 \mathrm{~cm}$ from the cathode where the substrate was used to be located. The diode laser was used for the argon $1 s_{5}-2 p_{9}$ transition (in Paschen's notation) with a center wavelength of $811.53 \mathrm{~nm}$ [19]. Measurements were carried out at sufficiently low laser powers where saturation effects are negligible $(<5 \%)$.

The laser diode scan frequency was fixed at $0.5 \mathrm{~Hz}$, so that during one half period of the wavelength scan 100 plasma pulses may occur. Three channels were recorded into the oscilloscope: the etalon signal (black curve, figure 1a), the absorption signal (blue curve, figure 1a), and the trigger signal of Ti magnetron (red curve, Fig. 1a). The absorption signal was first subtracted by the offset, then rescaled from time scale into frequency scale using etalon signal as reference. The rescaled absorption signal was subsequently be reconstructed with regard to the triggering of magnetron to form time resolved absorption profile of each time point in the discharge period as shown in figure $1 \mathrm{~b}$. From these time resolved absorption profile one can easily calculate the time resolved temperature and density of argon metastable using the analysing sequence described in [20].

\section{RESULTS AND DISCUSSIONS}

\section{III.1. Time resolvedargon metastable density and temperature in HiPIMS and in dual HiP- IMS}

The time resolved density and temperature of argon metastable in an original HiPIMS discharge with $\mathrm{Cu}$ as target material withthe same mean discharge current as the $\mathrm{Cu}$ pulse in the dual-HiPIMS were also measured to serve as the reference for further investigation. The resultis displayed in figure 2a. The argon metastable density features a double peak structure where the first maximum occurring during the pulse is followed by a second maximum in the afterglow. Both regimes are well separated from each other. The first maximum during the pulse is caused by electron impact excitation of argon atoms. The rapidly decreasing of metastable density in the afterglow after the plasma is switched off is largely caused by de-excitation throught electron 

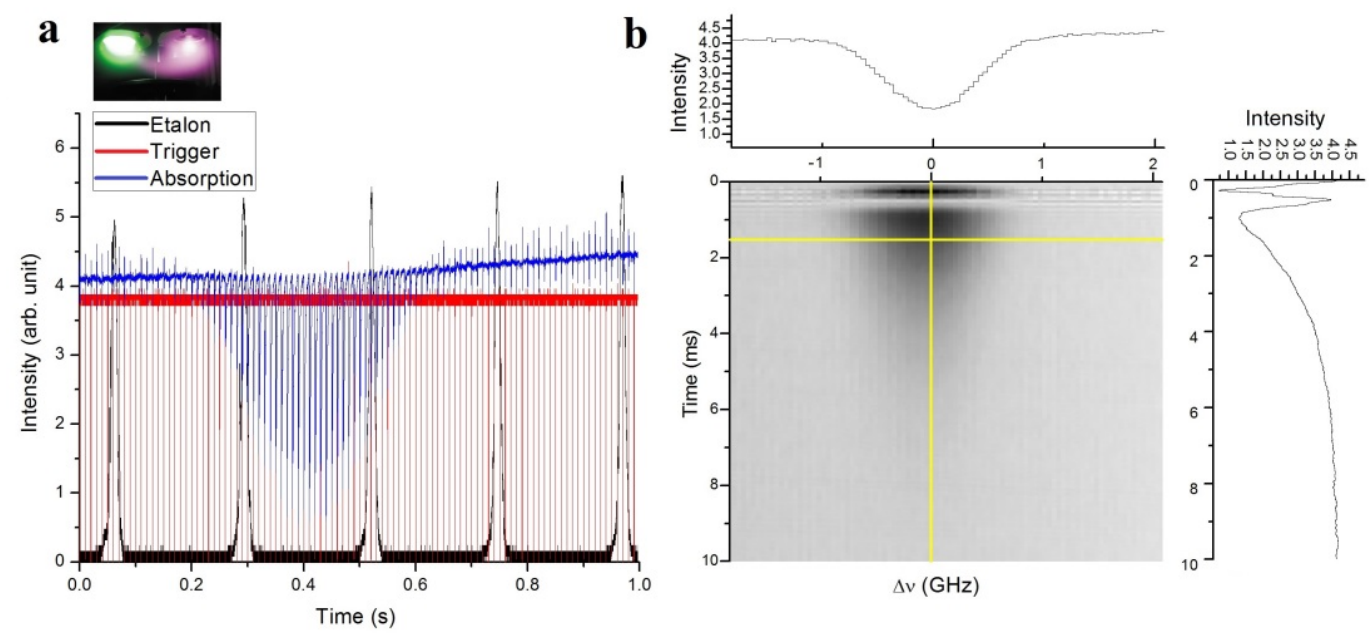

Fig. 1. Data acquisition (a) as measured, (b) reconstructed

collision. The following second maximum in the afterglow can be explained by electron-ion recombination giving rise to the formation of excited argon atoms (Ar*) which eventually decay to the metastable state [14,21].

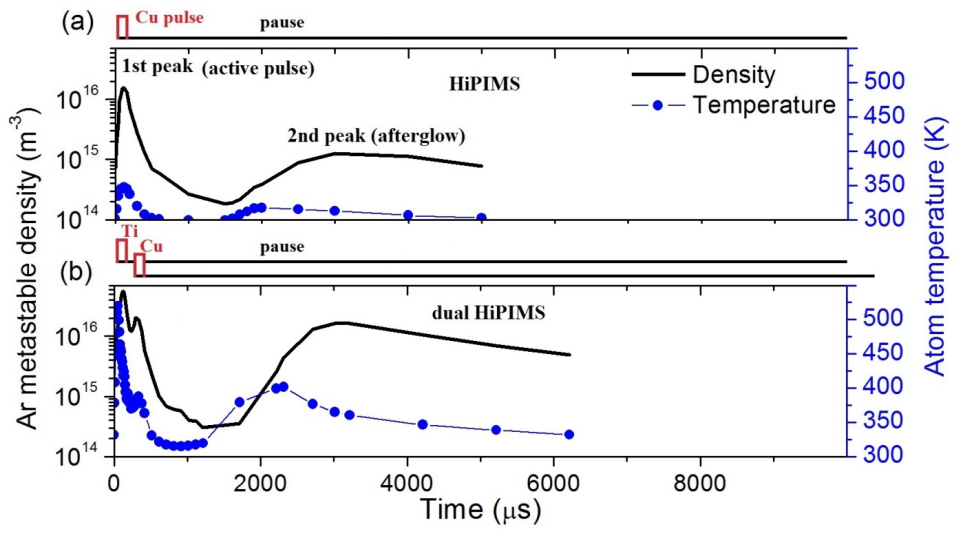

Fig. 2. Time resolved density and temperature of argon metastable in (a) HiPIMS discharges and (b)dual HiPIMS with $115 \mu$ s delay.

The time dependence of temperature is quite similar to the temporal evolution of the metastable density. The temperature displays a pronounced increase of about $50 \mathrm{~K}$ during the plasma on phase followed by a fast decrease in the afterglow and a subsequent moderate increase. The first rise correlates with the increase in the Ar metastable density; it is presumably caused by the heating of the plasma due to ions, fast neutralized argon ions reflected by the cathode, and the energy released by sputtered atoms. The second temperature rise during the afterglow reflects the ion recombination effect. The exact heating mechanism is not clear, however. It appears that ions 
Table 1. Dependence of plasma behavior (metastable density) on delay time

\begin{tabular}{cccc}
\hline Delay $(\boldsymbol{\mu} \mathbf{s})$ & $\begin{array}{c}\mathbf{1}^{\text {st }} \text { density } \\
\text { peak }\left(\mathbf{m}^{-3}\right)\end{array}$ & $\begin{array}{c}\mathbf{2}^{\text {nd }} \text { density } \\
\text { peak }\left(\mathbf{m}^{-\mathbf{3}}\right)\end{array}$ & $\begin{array}{c}\text { Density at the beginning } \\
\text { of the pulse }\left(\mathbf{m}^{-\mathbf{3}}\right)\end{array}$ \\
\hline HiPIMS & $1.80 \mathrm{e} 16$ & $1.27 \mathrm{e} 15$ & $2.3 \mathrm{e} 14$ \\
\hline 15 & $2.34 \mathrm{e} 16$ & $1.72 \mathrm{e} 16$ & $6.1 \mathrm{e} 16$ \\
\hline 115 & $2.04 \mathrm{e} 16$ & $1.67 \mathrm{e} 16$ & $1.4 \mathrm{e} 16$ \\
\hline 515 & $1.93 \mathrm{e} 16$ & $7.24 \mathrm{e} 15$ & $3.3 \mathrm{e} 16$ \\
\hline 1015 & - & $2.70 \mathrm{e} 15$ & $8.0 \mathrm{e} 16$ \\
\hline 2015 & - & $1.45 \mathrm{e} 15$ & $4.6 \mathrm{e} 14$ \\
\hline 3015 & - & $1.54 \mathrm{e} 15$ & $2.9 \mathrm{e} 16$ \\
\hline
\end{tabular}

are not fully thermalized and, as a consequence, the metastable atoms forming from these ions by recombination thus attain a higher temperature compared with the surrounding gas atoms.

The argon metastable density and temperature of $\mathrm{Cu}$ pulse in a dual HiPIMS discharge qualitatively behaves almost similar to that of an ordinary HiPIMS discharge. However, there are quantitatively differences owning to the influence of the Ti pulse on the $\mathrm{Cu}$ pulse. As can be seen in Fig. 2, in dual HiPIMS discharge both first density and temperature peaks are slightly higher than in HiPIMS discharge, meanwhile both second peaks in the former are significantly higher than those in the later.

\section{III.2. Influence of pre-ionization effectfrom Ti pulse on the plasma behavior}

\section{a) Metastable density}

There is clearly a positive influence of Ti pulse on $\mathrm{Cu}$ pulse in a dual HiPIMS system as the corresponding peak density in dual HiPIMS is always higher than that of the ordinary one (see Table 1). There is no double the influence of the pre-ionization effect which manifests itself in an elevation of $\mathrm{Cu}$ peak density of about $30 \%$ for $15 \mu$ s delay. The first peak density decreases toward the peak density in HiPIMS discharge with longer delay as the pre-ionization effect would become smallersince the plasma density decreases exponentially in the afterglow [13].

The $2^{\text {nd }}$ peak density also shows the same behavior (see Table 2), however, the influence of Ti pulse is much stronger. The peak density is $1.72 \times 10^{16} \mathrm{~m}^{-3}$ for $15 \mu$ s delay which is 13 times higher than that in HiPIMS. This density decreases exponentially toward the value of that in HiPIMS discharge. This strong enhancement of the $2^{\text {nd }}$ peak density due to pre-ionization effect is understandable. As has been pointed out in equation 17 in [14], the production of metastable in the afterglow is proportional to electron density and inversely proportional to electron temperature. This production will be significantly stronger for strongerpre-ionization effect owning to the increase of electron density and the simultaneously decrease of electron temperature from this effect.

The behavior of peak densities with respect to the change of time delay implies that the preionization effect from the previous pulse promotes the subsequence pulse which result in a stronger plasma behavior. The pre-ionization effect decreases drastically as the delay time prolongs. 


\section{b) Atom temperature}

Fig. 3 shows the temporal behavior of metastable atom temperature in the $\mathrm{Cu}$ pulse for different delay times in comparison to the original HiPIMS (the red curve).In line with what we have found in metastable density, the metastable atom temperature peak in dual HiPIMS plasma is higher for shorter delay and always higher than that in HiPIMS. The higher metastable atom temperature peak reflects a stronger ion bombardment current $\left(\mathrm{Ar}^{+}\right)$into the target which eventually creates more hot metastable atom. Therefore one would expect a higher plasma density of the subsequence pulse under the influence of pre-ionization effect which support the result from Langmuir probe measurement in our previous work [13]. As the delay prolonged the effect vanishes and the pulse resumes its behavior in an original HiPIMS discharge.
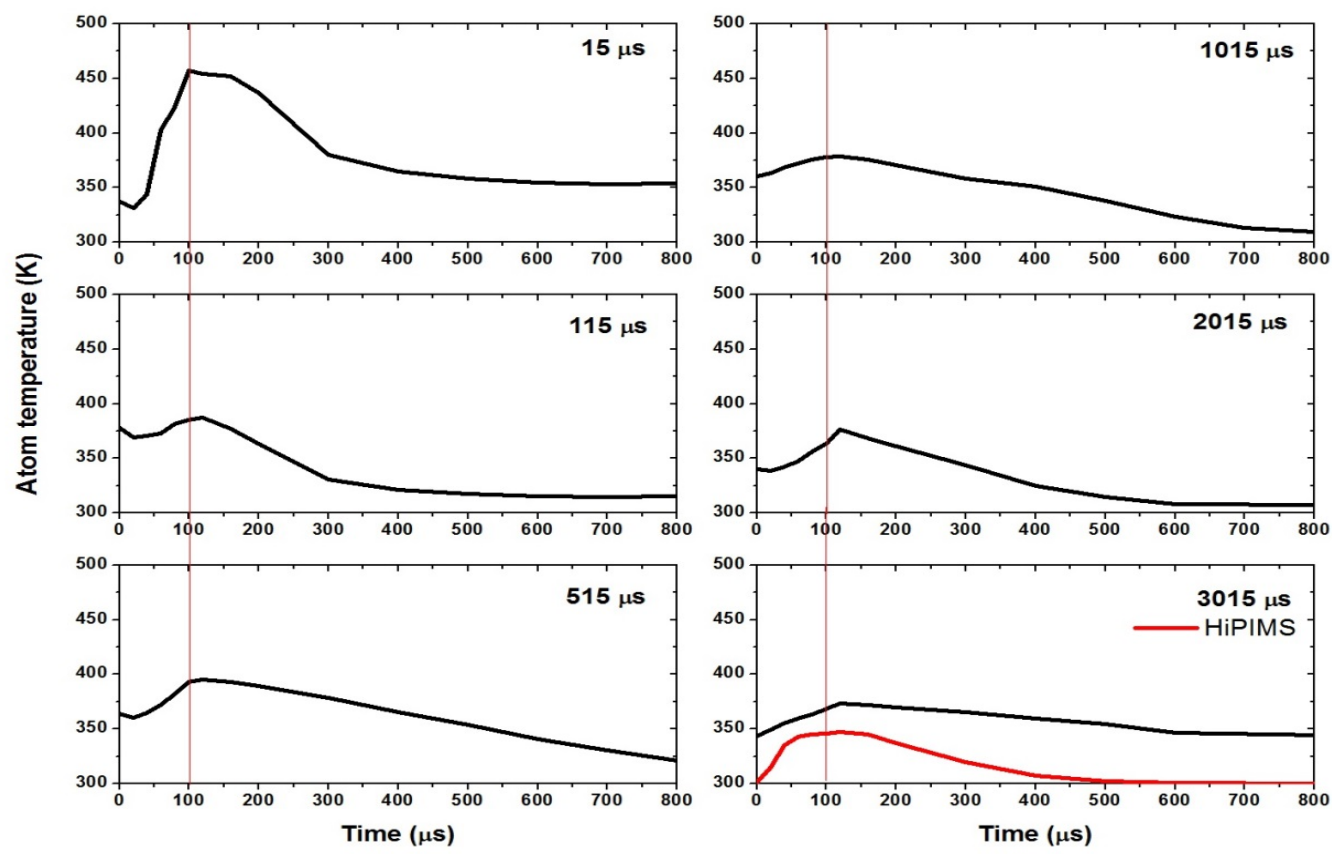

Fig. 3. Temporal behavior of metastable atom temeperature of $\mathrm{Cu}$ pulse with different delay times (delay of the $\mathrm{Cu}$ pulse to the Ti pulse). The red curvel displays the time revolution of metastable atom temperature in HiPIMS.

\section{III.3. Contribution of metastable atoms to the pre-ionization effect}

As can be seen in Table 1, there is no relation between the metastable density when the pulse started with its behavior. For example, with $1015 \mu$ s delay, the $\mathrm{Cu}$ pulse started at pretty high metastable density $\left(8 \times 10^{16} \mathrm{~m}^{-3}\right)$ but all its plasma properties are rather weak. One can conclude that, even though the ionization energy of metastable atoms is very small (in comparison to ground state) they do not contribute to the fast ignition of the pulse and of couse do not play any role in the pre-ionization effect. 


\section{CONCLUSIONS}

We have performed a time-resolved tunable diode laser absorption spectroscopy to investigate the pre-ionization effect in dual high power impulse magnetron sputtering discharges with two cathodes in a closed magnetic field configuration. The $\mathrm{Cu}$ pulse in a dual-HiPIMS feature almost similar behavior of density as well as temperature of metastable in a single HiPIMS. The preionization effect enhances plasma behavior of the subsequence pulse ( $\mathrm{Cu}$ pulse). However, this effect vanishes significantly as the delay was prolonged. Although metastable atoms are abundantly available in the afterglow, they play minor role in the ignition of the subsequence pulse.

\section{ACKNOWLEDGMENTS}

This work was supported by the National Foundation for Science and Technology Development (NAFOSTED), Vietnam, under project number 103.05-2012.20.

\section{REFERENCES}

[1] R. K. Waits, J. Vac. Sci. Technol. 15, 179 (1978).

[2] K. Ellmer, in Low Temperature Plasmas, edited by R. Hippler, H. Kersten, M. Schmidt, and K. H. Schoenbach (Wiley, New York, 2009), Vol. 2, p. 675.

[3] I. Petrov, P. B. Barna, L. Hultman, J. E. Greene, J. Vac. Sci. Technol. 21 (2003) 117.

[4] V. Stranak, M. Cada, M. Quaas, S. Block, R. Bogdanowicz, S. Kment, H. Wulff, Z. Hubicka, C. A. Helm, M. Tichy, R. Hippler, J. Phys. D: Appl. Phys. 42 (2009) 105204.

[5] J. Bohlmark, M. Lattemann, J. T. Gudmundsson, A. P. Ehiasarian, Y. A. Gonzalvo, N. Brenning, and U. Helmersson, Thin Solid Films 515 (2006) 1522.

[6] U. Helmersson, M. Lattemann, J. Bohlmark, A. P. Ehiasarian, and J. T. Gudmundsson, Thin Solid Films 513 (2006) 1.

[7] K. Sarakinos, J. Alami, and S. Konstantinidis, Surf. Coat. Technol. 204 (2010) 1661.

[8] J. Vlček, A. D. Pajdarov, and J. Musil, Contrib. Plasma Phys. 44 (2004) 426.

[9] A. Anders, J. Andersson, and A. Ehiasarian, J. Appl. Phys. 102 (2007) 113303.

[10] A. Aijaz, D. Lundin, P. Larsson, and U. Helmersson, Surf. Coat. Technol. 204 (2010) 2165.

[11] J. Musil, P. Baroch, J. Vlcek, K. H. Nam, and J. G. Han, Thin Solid Films 475 (2005) 208.

[12] A. Bogaerts, E. Bultinck, I. Kolev, L. Schwaederl, K. V. Aeken, G. Buyle, and D. Depla, J. Phys. D: Appl. Phys. 42 (2009) 194018.

[13] V. Stranak, S. Drache, M. Cada, Z. Hubicka, M. Tichy, and R. Hippler, Contrib. Plasma Phys. 51(2-3) (2011) $237-245$

[14] V. Sushkov, H. T. Do, M. Cada, Z. Hubicka and R. Hippler, Plasma Sources Sci. Technol. 22 (2013) 015002.

[15] C. Vitelaru, D. Lundin, G. D. Stancu, N. Brenning, J. Bretagne and T. Minea, Plasma Sources Sci. Technol. 21 (2012) 025010.

[16] V. Stranak, M. Cada, Z. Hubicka, M. Tichy, R. Hippler, J. Phys. D: Appl. Phys. 108 (2010) 043305

[17] V. Stranak, M. Quaas, H. Wulff, Z. Hubicka, S. Wrehde, M. Tichy, R. Hippler, J. Phys. D: Appl. Phys. 41 (2008) 055202.

[18] S. Pfau, M. Tichy, Langmuir probe diagnostics; in: Low Temperature Plasma Physics, R. Hippler, H. Kersten, M. Schmidt, K.-H. Schoenbach, Eds., Wiley-VCH, Berlin, 175 (2008).

[19] Y. Ralchenko, A. E. Kramida, J. Reader and NIST ASD Team 2011 NIST Atomic Spectra Database (ver. 4.1.0) (Gaithersburg, MD: NIST) http://physics.nist.gov/asd

[20] H. T. Do, V. Sushkov and R. Hippler, New Journal of Physics 11 (2009) 03302.

[21] A. Bogaerts, J. Anal. At. Spectrom. 22, 502 (2007). 